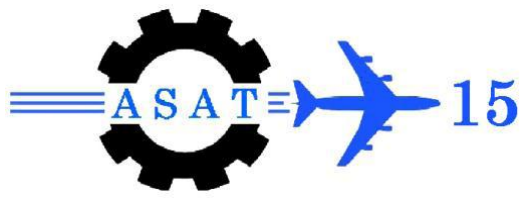

\title{
Aeroelastic Investigation of Composite Plate Wing in Subsonic Flow
}

\author{
M. Haidar ${ }^{*}$, M. A. Kamel ${ }^{\dagger}$, A. M. El Shabka ${ }^{\dagger}$, H. M. Negm ${ }^{\ddagger}$
}

\begin{abstract}
Aeroelastic phenomena including divergence and flutter are investigated for composite plate wing without sweep angle. Suitable derivations of aerodynamic loads have been developed for calculating both flutter speed $\left(U_{F}\right)$ and divergence speed $\left(U_{D}\right)$. Equivalent plate method is used for structural modeling of composite plate wing. Approximate solutions from the linearized formulation are obtained for studying steady and unsteady aerodynamic loadings on harmonically oscillating wing in subsonic flow. The wing is divided into element panels on which lift distributions are represented by concentrated lift forces. The pressure coefficient distribution is calculated at root chord using steady and unsteady aerodynamic modeling. The span wise distribution of lift coefficient is calculated from the pressure coefficient. The numerical results for both $\mathrm{U}_{\mathrm{F}}$ and $\mathrm{U}_{\mathrm{D}}$ show good agreement with previous works. Finally a parametric study of the effect of composite fiber's orientation on both $U_{F}$ and $\mathrm{U}_{\mathrm{D}}$ is carried out.
\end{abstract}

Keywords: Composite plate; flutter; divergence; unsteady aerodynamics.

\section{Introduction}

Flutter and divergence phenomena are defined respectively as dynamic and static instability of an aeroelastic system, characterized by the interaction of elastic deformation and aerodynamic loads. They are aeronautically very important since the onset of flutter/divergence may quickly develop into catastrophic structural failure or undesirable limit-cycle oscillations. Employing composite materials in the manufacture of aircraft structures has been one of the avenues to enlarge the flutter/ divergence boundary of an aircraft. This is due to the prominent characteristics associated with composite materials, namely, the high strength/stiffness-to-weight ratio and the controllable material properties that usually are not possessed by conventional materials. There are many methods used for structural modeling to study the aeroelastic phenomena of aircraft wings. One of these methods is the equivalent plate method [1].

Equivalent plate analysis has been used to replace the computationally expensive finite element analysis in initial design stages or in conceptual design of aircraft wing structures. In equivalent plate modeling, the model characteristics are represented with polynomials, which require only a small fraction of the input data that would be required by a corresponding finite element model. An equivalent plate analysis procedure based on Ritz method was proposed at NASA Langley Research Center as early as 1986. In the Ritz method-based equivalent plate

Syrian Armed Forces, Syria.

$\dagger \quad$ Egyptian Armed Forces, Egypt.

* Prof. Cairo University, Egypt. 
theory, the aircraft wing structure is modeled with several trapezoidal segments [2]. The plate wing is subjected to aerodynamic load; the values of steady and unsteady aerodynamic load can be calculated using steady and unsteady aerodynamic models.

Aerodynamic calculations constitute the second half of aeroelasticity problems because it is essential to know how wing deformations affect aerodynamic loads. The central problem in steady state aeroelasticity is the effect of elastic deformation on the lift distribution over lifting surface such as airplane wings and tails. At lower speeds of flight, the effect of elastic deformation is small. But at higher speeds of flight, the effect of elastic deformation may become so serious as to cause a wing to be unstable, or to render a control surface ineffective, or even to reverse the sense of control [3]. Although aerodynamic loads calculations change with the flight speed, only the incompressible flight regime is considered in the present work.

Divergence is a static aeroelastic effect that involves the interaction of aerodynamic and elastic forces. Wing torsional divergence is the most common divergence that occurs where the wing local angle of attack increases to the point where structural failure occurs. This happens when the lifting forces on a wing induce a torsional moment that twists the wing about its elastic axis. This twist results in an increase of the wing local angle of attack. This in turn produces more lift, which in turn induces further twist. Eventually, the aerodynamic forces and the elastic reactive forces will either reach equilibrium, or the reactive forces will be overcome. The local angle of attack will continue to increase, as will the aerodynamic forces, until structural failure occurs. Thus precisely at the divergence speed, the increase in aerodynamic torsional moment due to an increment in twist angle is equal to the corresponding increment in elastic restoring torque. If the aircraft exceeds this speed, the aerodynamic moment increase will be greater than the elastic restoring torque. Divergence speed is primarily affected by the coupling between the torsional and bending degrees of freedom. Thus, swept back wings are less susceptible to divergence than forward swept wings [4].

Flutter is generally observed on wings and control surfaces since these types of structures are subjected to large aerodynamic loads. Flutter is produced due to forces that are generated from the dynamic deflections of an elastic structure from the undeformed state. Flutter is defined by a critical speed $U_{F}$ and critical frequency $\omega_{F} . U_{F}$ is the lowest air speed and $\omega_{F}$ is the corresponding critical frequency at which a given structure flying at a given atmospheric condition will undergo sustained, simple harmonic oscillations. The solution of flutter leads to a complex eigenvalue problem where two characteristic numbers determine the speed and frequency.

Motivated by the problem of aeroelasticity, the present work presents the aeroelastic calculations of composite plate wing. The equivalent laminate plate modeling is used to calculate the stiffness and mass matrices for plate wing. The aerodynamic matrices are calculated using the doublet point method for both steady and unsteady aerodynamic loadings in subsonic flow. Matlab programs were prepared to calculate divergence speed, flutter speed, and aerodynamic characteristics. The divergence speed $U_{D}$ and flutter speed $U_{F}$ are calculated for a rectangular composite plate wing for different cases of composite fiber orientations. The results of aeroelastic calculations are compared with other analysis and experiments. 


\section{Equivalent Plate Modeling of Wing}

Using the First Order Shear Deformation Plate Theory (FSDPT) [5], the wing displacements in the $\mathrm{x}, \mathrm{y}$ and $\mathrm{z}$ directions respectively are approximated by:

$$
\begin{aligned}
& u(x, y, z, t)=u_{0}(x, y, t)-z \psi_{x}(x, y, t) \\
& v(x, y, z, t)=v_{0}(x, y, t)-z \psi_{y}(x, y, t) \\
& w(x, y, z, t)=w_{0}(x, y, t)
\end{aligned}
$$

These expressions includes five different unknown functions: $\mathrm{u}_{0}(\mathrm{x}, \mathrm{y}, \mathrm{t}), \mathrm{v}_{0}(\mathrm{x}, \mathrm{y}, \mathrm{t})$, $w_{0}(x, y, t), \psi_{x}(x, y, t)$ and $\psi_{y}(x, y, t)$ for the deformations field of the plate.

The Ritz method is used to obtain an approximate solution for the generalized displacements that minimize the total energy of the wing box structure. The total energy $\mathrm{E}_{\text {tot }}$ associated with the wing model is

$\mathrm{E}_{\mathrm{tot}}=\mathrm{U}+\mathrm{W}-\mathrm{T}$

where $\mathrm{U}$ is the potential energy stored in the structure through deformation, $\mathrm{W}$ is the work of the applied loads moving through the corresponding structural deflections, and $\mathrm{T}$ is the kinetic energy associated with the mass of the structure. All the equations used for calculating the stiffness and mass matrices of composite plate wing are presented in [5].

\section{Aerodynamic Modeling}

Unsteady aerodynamics is a branch of science which studies the motion of the profile or the wing in a free stream when it changes by time, and so do the acting aerodynamic coefficients.

When the changes in the motion are fast enough, the aerodynamic response of the body will have a phase lag. For fast changes in the motion, the inertia of the displaced air will contribute as the apparent mass term. If the apparent mass term is negligible, this type of analysis is called quasi-unsteady aerodynamics [6]. An important problem for aeroelasticians is to evaluate the pressure distribution on a wing in oscillatory motion. Many methods have been developed for calculating the unsteady pressure distribution on a thin finite wing in subsonic flow, one of these methods is the doublet point method (DPM).

The Doublet point method is based on a concept of concentrated lift forces. The wing is divided into element surface panels on which lift distributions are represented by single concentrated lift forces. Since the procedure does not include any quadratures, it can be applied easily to calculate the unsteady aerodynamic loadings on complex planform wings even when they have partial span control surfaces. Amplitudes of pressure distributions on oscillatory lifting surfaces and of their upwash velocity $(v)$ are related by the following integral equation [7]:

$$
v(x, y)=\frac{1}{8 \pi} \iint_{S} \Delta P(\xi, \eta) \cdot K\left(x_{0}, y_{0}\right) d \xi d \eta
$$

where,

S: denotes the region of the wing area

$\Delta P:$ a pressure coefficient $\Delta P=\frac{-P_{+}^{\prime}-P_{-}^{\prime}}{1 / 2 \rho_{\infty} U^{2}}$ 
$P_{+}^{\prime}, P_{-}^{\prime}$ : disturbance pressure on upper and lower surfaces

$\mathrm{K}$ : Kernel function

The wing planform is divided into panel segments called element surfaces as shown in Fig.(1). The trapezoid of the element has the area $\Delta_{i}$ and width $2 \sigma_{i}$. The (1/4-3/4) chord rule is adopted for element surfaces as shown in Fig (2). The lift distribution on the surface is concentrated at the point $\left(\xi_{\mathrm{i}}, \eta_{\mathrm{i}}\right)$ on the quarter chord at the midspan of the element. This is equivalent to putting a doublet source of strength $\Delta P\left(\xi_{i}, \eta_{i}\right) . \Delta_{i}$. Thus the location $\left(\xi_{\mathrm{i}}, \eta_{\mathrm{i}}\right)$ is the doublet point of the element surface. Similarly, the upwash of the three-quarter chord point $\left(\mathrm{x}_{\mathrm{i}}, \mathrm{y}_{\mathrm{i}}\right)$ at midspan is taken as representative for the whole upwash distribution on an element surface.

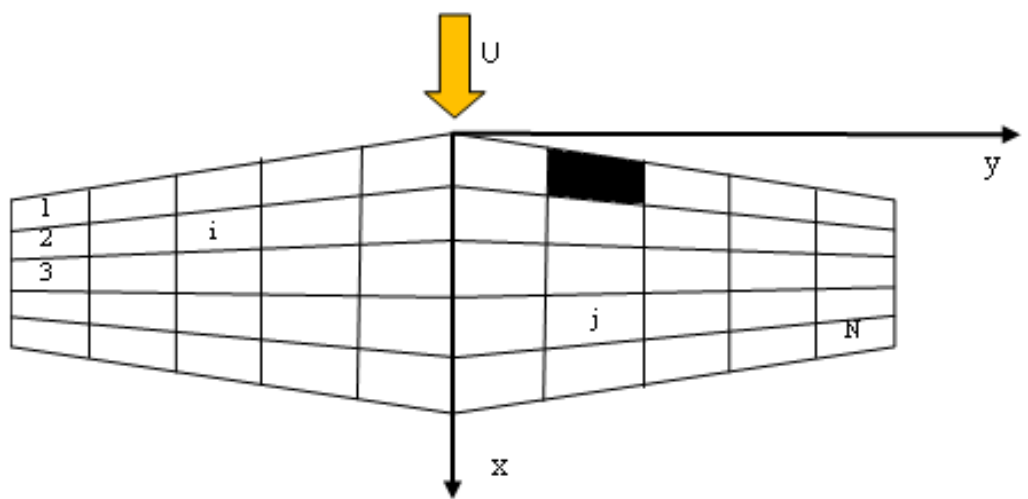

Fig(1) Element surfaces

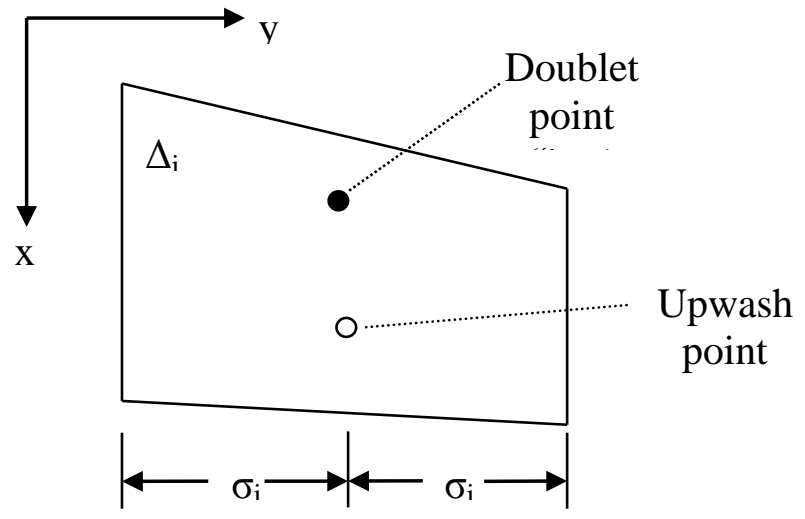

Fig(2) The ith element surface

The upwash $\mathrm{w}_{\mathrm{i}}$ of the $i$ th element can be calculated in a discrete system by

$v_{i}=\frac{1}{8 \pi} \sum_{j=1}^{N} \Delta P\left(\xi_{j}, \eta_{j}\right) \cdot \Delta_{j} \cdot K\left(x_{i}-\xi_{j}, y_{i}-\eta_{j}\right) ; i=1,2, \ldots, N$

By introducing the following relations:

$[D]=\left[d_{i j}\right]=\frac{\Delta_{j}}{8 \pi} K\left(x_{i}-\xi_{j}, y_{i}-\eta_{j}\right)$ 


$$
\begin{aligned}
& \{P\}=\left\{P_{j}\right\}=\left\{\Delta P\left(\xi_{j}, \eta_{j}\right)\right\} \\
& \{v\}=\left\{v_{i}\right\}=\left\{v\left(x_{i}, y_{i}\right)\right\}
\end{aligned}
$$

Then the following algebraic equation is obtained

$$
\{v\}=[D] \cdot\{P\}
$$

where: [D] aerodynamic matrix

The upwash vector $\mathrm{W}$ is calculated from the given oscillatory mode shape $\mathrm{h}(\mathrm{x}, \mathrm{y})$ by

$v_{i}=\frac{\partial}{\partial x} h\left(x_{i}, y_{i}\right)+i k h\left(x_{i}, y_{i}\right)$

where:

$h=\bar{h} \cdot e^{i \omega t}$ The vertical translation in $\mathrm{z}$ direction

$k$ Reduced frequency

$\omega$ The oscillation frequency

The complex lift coefficient is defined for every element surfaces $\mathrm{C}_{l}$ as:

$$
C_{l}\left(y_{i}\right)=\frac{\sum_{i}^{N x} \Delta P_{i} \Delta_{i}}{\sum_{i}^{N x} \Delta_{i}}
$$

The doublet point method was applied for a rectangular wing with low aspect ratio $(A R=2)$, and the number of elements in $\mathrm{x}, \mathrm{y}$ directions are $\mathrm{Nx}$, $\mathrm{Ny}$ respectively. Spanwise lift distribution for plate was calculated. The obtained results show good agreement with Morino results presented in [8] as shown in Fig (3).

Figure (4) shows good agreement of calculated chordwise pressure distribution at plate root compared with Morino results presented in [8].

Figure (5) shows the 3-D chordwise and spanwise pressure distribution (real and imaginary parts)

\section{Aeroelastic Analysis}

The structural model of the composite plate wing is obtained using equivalent laminate plate method.

\subsection{Flutter analysis}

The governing equation of harmonic motion of the wing takes the form

$$
[K]\{q\}+[M]\{\ddot{q}\}=\{F\}
$$

where:

[K] Stiffness matrix

[M] Mass matrix 
$\{q\}$ Displacement vector function of time (t)

$\{F\}$ Aerodynamic force vector

In harmonic motion: $q=\bar{q} e^{i \omega t}$

So the governing equation can be obtained by assembling the elements as:

$[K] .\{q\}-\omega^{2}[M]\{q\}=\{F\}$
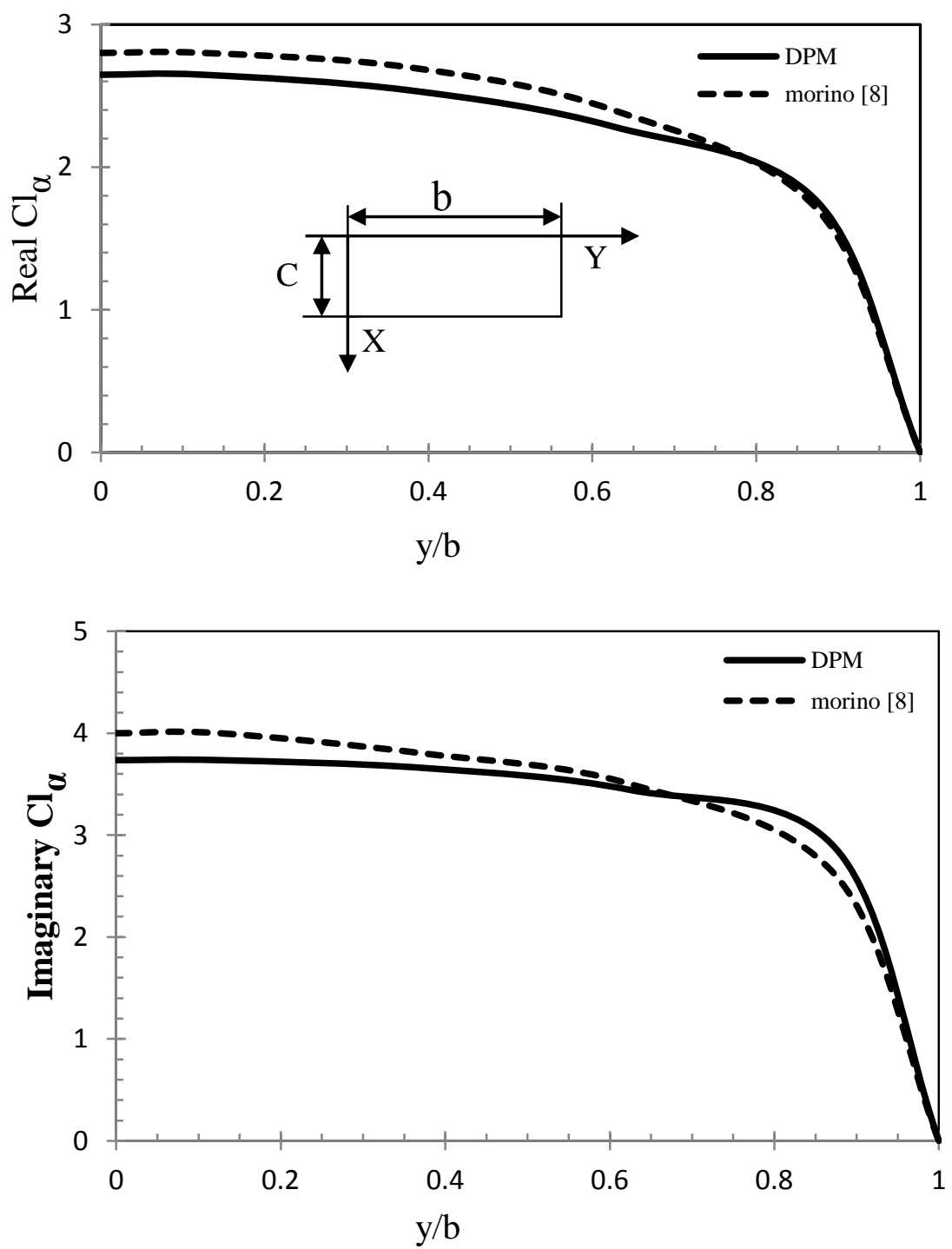

Fig (3) Spanwise lift distribution $(\mathrm{AR}=2, \mathrm{k}=1, \mathrm{M}=\mathbf{0}, \mathrm{Nx}=6, \mathrm{Ny}=4)$

The concentrated forces at doublet points are given by:

$[F]=\frac{1}{2} \rho U^{2}[\Delta] .\{P\}$ 
$\{P\}$ is the pressure coefficient distribution vector which can be calculated using the doublet point method as given by Eq. (8) :

$\{P\}=[D]^{-1}\{v\}$

where:

$[\Delta]$ area matrix

$\{v\}$ upwash vector (normal wash vector)
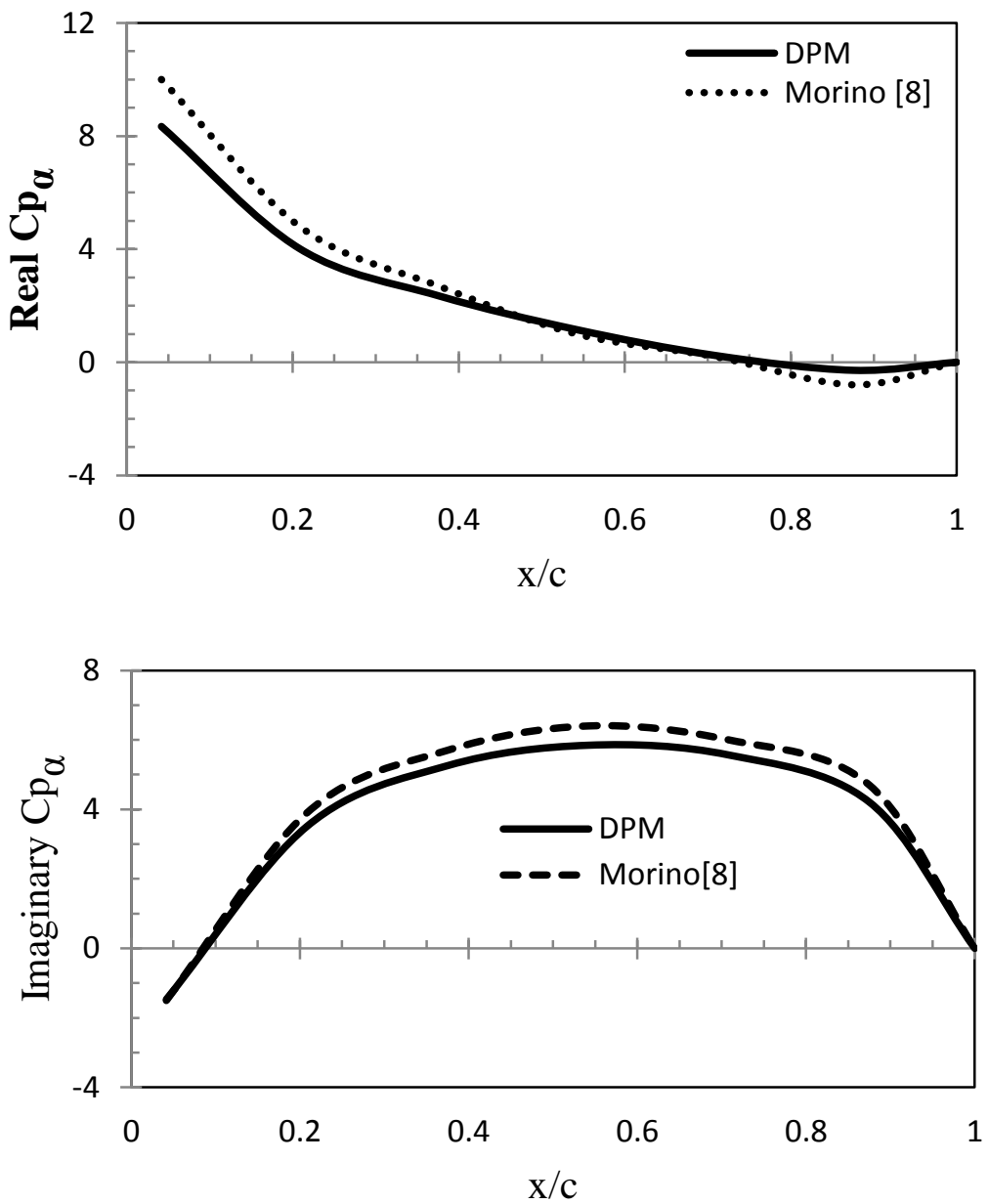

Fig(4) Chordwise Pressure coefficient distribution $(\mathrm{AR}=2, \mathrm{k}=1, \mathrm{M}=0, \mathrm{Nx}=6, \mathrm{Ny}=5)$ 

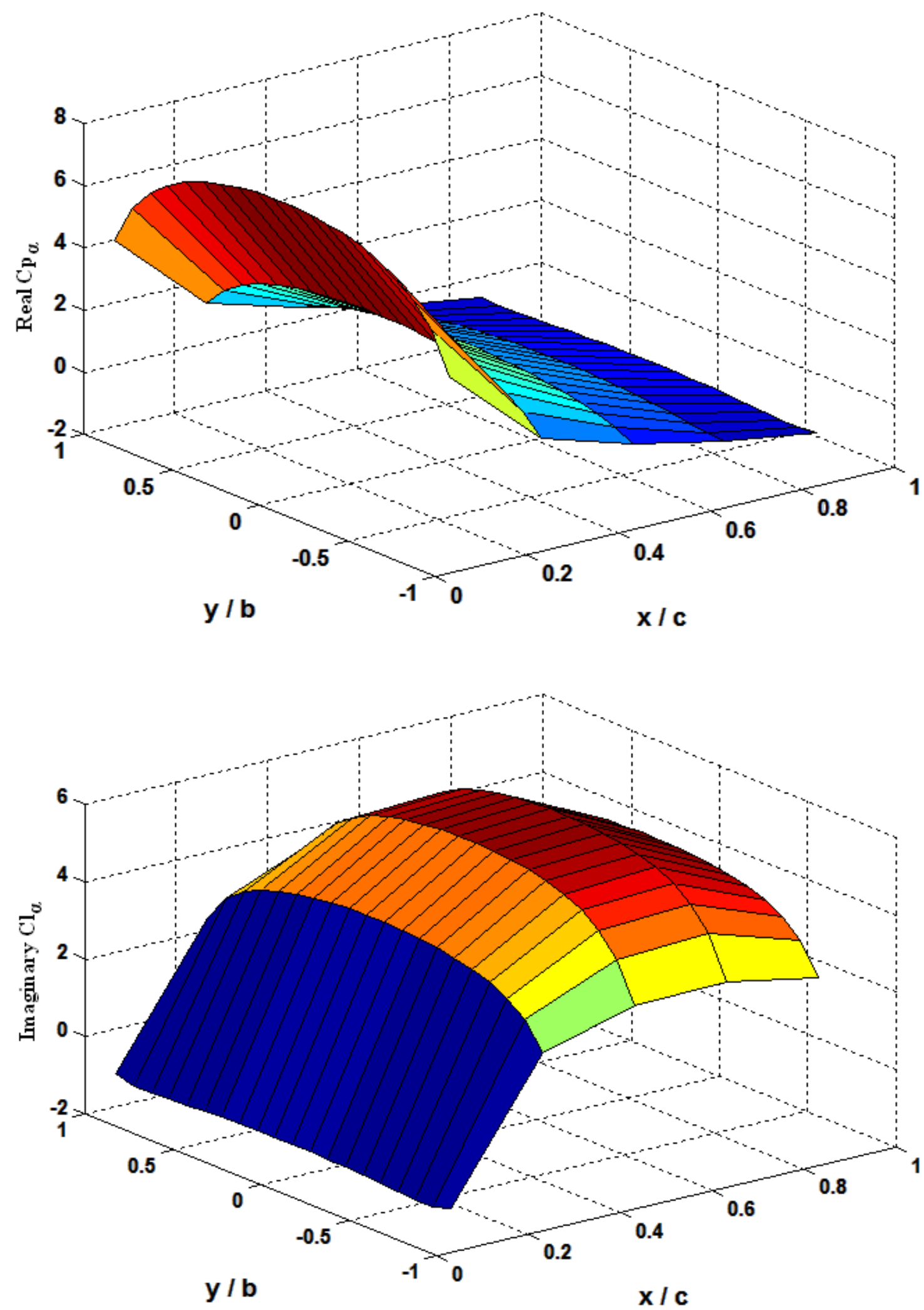

Fig (5) Chordwise and Spanwise Pressure distribution $(\mathrm{AR}=2, \mathrm{k}=1, \mathrm{M}=0, \mathrm{Nx}=6, \mathrm{Ny}=5)$ 
To get the upwash vector as a function of the unknown Ritz coefficients [1], the nondimensionalized upwash velocity in pitching oscillation motion about middle chord line $\mathrm{W}$ is given by:

$v_{i}=\frac{\partial}{\partial x} h\left(x_{i}, y_{i}\right)+i \frac{k}{\bar{c}} h\left(x_{i}, y_{i}\right)$

$h\left(x_{i}, y_{i}\right)$ is the deformation at the upwash point $\left(x_{i}, y_{i}\right)$

$\bar{c}$ is semichord

For a cantilever plate fixed along one side we take the following polynomial in the $\mathrm{z}$ direction which satisfied boundary conditions $(\mathrm{y}=0 \Rightarrow h=0)$ :

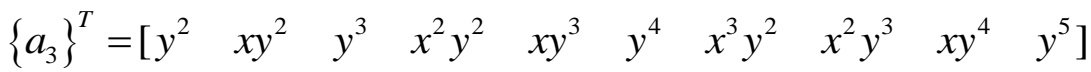

From Ritz approximate function:

$h\left(x_{i}, y_{i}\right)=\left\{a_{3}\left(x_{i}, y_{i}\right)\right\}^{T} \cdot\left\{q_{3}\right\}$

where: $\mathrm{q}_{3}$ is displacement vector in $\mathrm{z}$ direction

The upwash vector in matrix form is:

$\{v\}=\left(\left[v_{R}\right]+i\left[v_{I}\right]\right) \cdot\left\{q_{3}\right\}$

According to Eq. (13), the concentrated force vector using equivalent plate model will be:

$\{F\}=\frac{1}{2} \rho U^{2}[\Delta][D]^{-1}\{v\}\left\{q_{3}\right\}$

where:

$\mathrm{U}$ : free stream velocity

Since the reduced frequency

$k=\frac{\bar{c} \omega}{U}$

Then

$U=\frac{\omega \bar{c}}{k}$

Finally

$\{F\}=\omega^{2} \cdot\left[Q^{*}\right] \cdot\left\{q_{3}\right\}$

where

$\left[Q^{*}\right]=\frac{\rho b^{2}}{2 k^{2}} \cdot[\Delta] \cdot[D]^{-1} \cdot[v]$

Substituting into Eq. (12) we get the equation of motion: 


$$
\left[[K]-\omega^{2}\left([M]+\left[Q^{*}\right]\right)\right]\left\{q_{3}\right\}=0
$$

Solving the eigenvalue problem

$\left|[K]-\omega^{2}\left([M]+\left[Q^{*}\right]\right)\right|=0$

gives

$\omega^{2}=\operatorname{Re}\left(\omega^{2}\right)+\operatorname{Im}\left(\omega^{2}\right)$

$\omega=\sqrt{\operatorname{Re}\left(\omega^{2}\right)} \quad$ Flutter frequency or natural frequency according to the value of $\mathrm{k}$

$g=\frac{\operatorname{Im}\left(\omega^{2}\right)}{\operatorname{Re}\left(\omega^{2}\right)} \quad$ Damping

\subsection{Divergence analysis}

The aerodynamic force due to steady aerodynamic pressure is:

$\{F\}=\frac{1}{2} \rho U^{2}[\Delta][A]^{-1}\{v\}\left\{q_{3}\right\}$

$[A]$ is steady aerodynamic matrix

$\{F\}=U^{2} \cdot\left[Q^{*}\right] \cdot\left\{q_{3}\right\}$

where

$$
\left[Q^{*}\right]=\frac{1}{2} \cdot \rho \cdot[\Delta] \cdot[A]^{-1} \cdot\{v\}
$$

Then

$$
[K] \cdot\{q\}=U^{2}\left[Q^{*}\right]\left\{q_{3}\right\}
$$

$\left[\frac{1}{U^{2}}[K]-\left[Q^{*}\right]\right]\left\{q_{3}\right\}=0$

$\frac{1}{U^{2}}=\lambda$

$\left[\lambda[K]-\left[Q^{*}\right]\right]\left\{q_{3}\right\}=0$

The largest positive eigenvalue $\lambda$ gives the lowest speed (divergence speed)

$U_{D}=\sqrt{\frac{1}{\lambda_{\max }}}$

\section{Numerical Investigation and Results}

Divergence and flutter speeds of a composite rectangular plate wing are presented. The plate has semispan "b" of $0.3 \mathrm{~m}$, chord "a" of $0.1 \mathrm{~m}$ and thickness of $1.5 \times 10^{-3} \mathrm{~m}$ as shown in Fig (5). The plate is made of three layers of HMS/DX 210 composite material of the properties: 


$$
\begin{array}{ll}
\mathrm{E}_{1}=1.727 * 10^{11} & {\left[\mathrm{~N} / \mathrm{m}^{2}\right]} \\
\mathrm{E}_{2}=7.2 * 10^{9} & {\left[\mathrm{~N} / \mathrm{m}^{2}\right]} \\
\mathrm{G}_{12}=3.76^{*} 10^{9} & {\left[\mathrm{~N} / \mathrm{m}^{2}\right]} \\
\mathrm{G}_{23}=2.35 * 10^{9} & {\left[\mathrm{~N} / \mathrm{m}^{2}\right]} \\
\mathrm{G}_{31}=\mathrm{G}_{12} & {\left[\mathrm{~N} / \mathrm{m}^{2}\right]} \\
\nu=0.3 & \\
\mathrm{t}_{\mathrm{ply}}=0.5 * 10^{-3} & {[\mathrm{~m}]} \\
\rho=1550 & {\left[\mathrm{Kg} / \mathrm{m}^{3}\right]}
\end{array}
$$
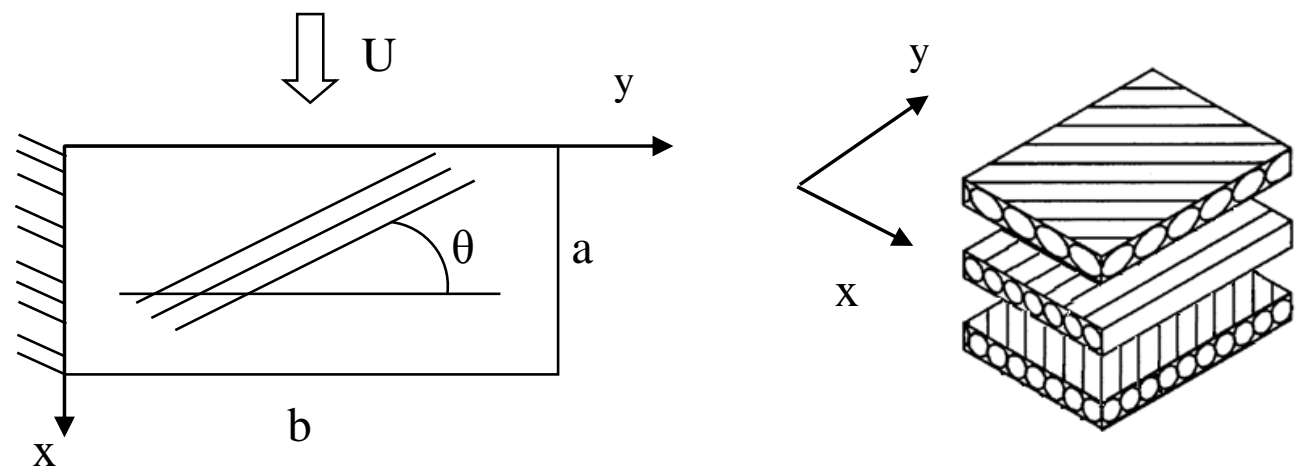

Fig (5) Plate wing and stacking sequence

The divergence and flutter speeds are calculated for the composite plate where its layers have the symmetric stacking sequence $[\theta / 0 / \theta]$. Fig (6) shows variation of the divergence speed vs. ply angle $\theta$ as compared with the result given in ref [9]. There is no divergence at the positive angles between $20^{\circ}$ up to $70^{\circ}$, while at negative angles; the divergence speed is nearly constant up to $-10^{\circ}$. The divergence speed increases rabidly as the angle increases above zero.

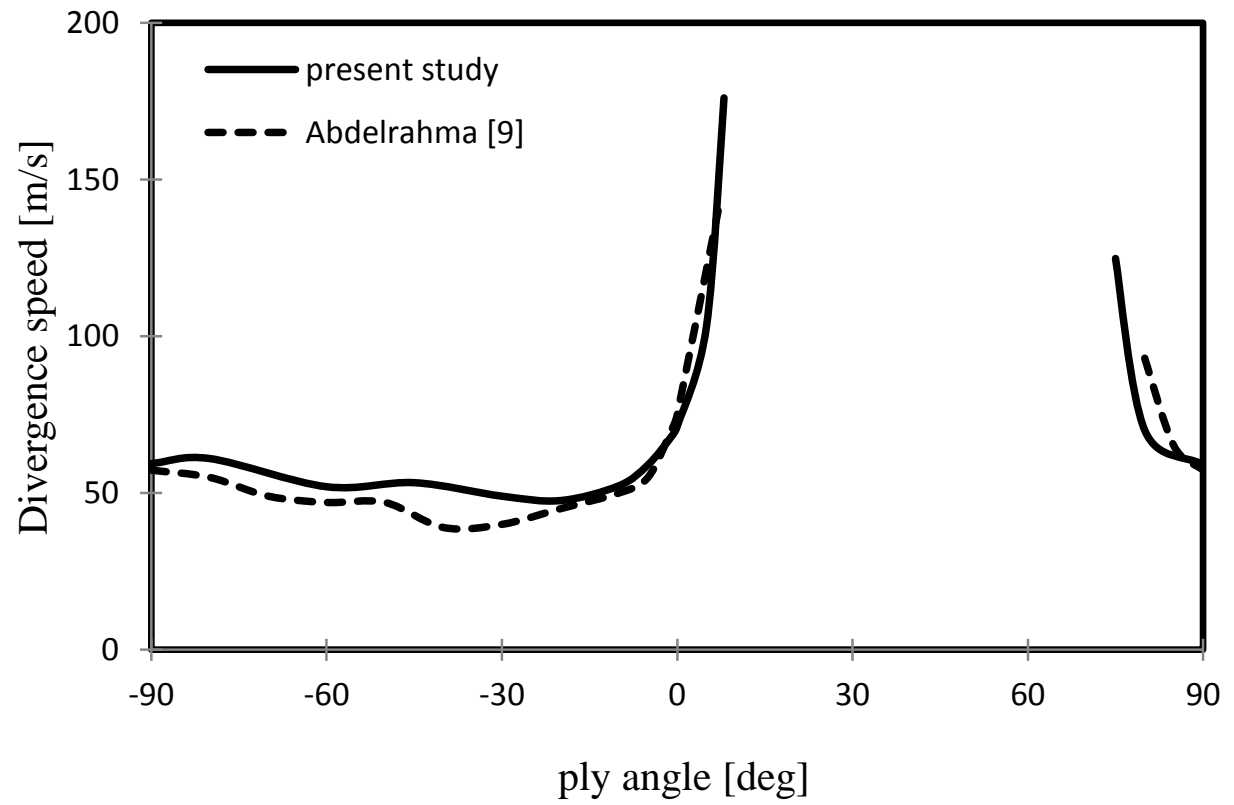

Fig (6) Variation of divergence speed with ply angle for a $[\theta / 0 / \theta]$ rectangle wing plate 
Figure (7) shows variation of the flutter speed vs. ply angle as compared with the results given in ref [10]. $\pm 45^{\circ}$ give highest values of the flutter speed.

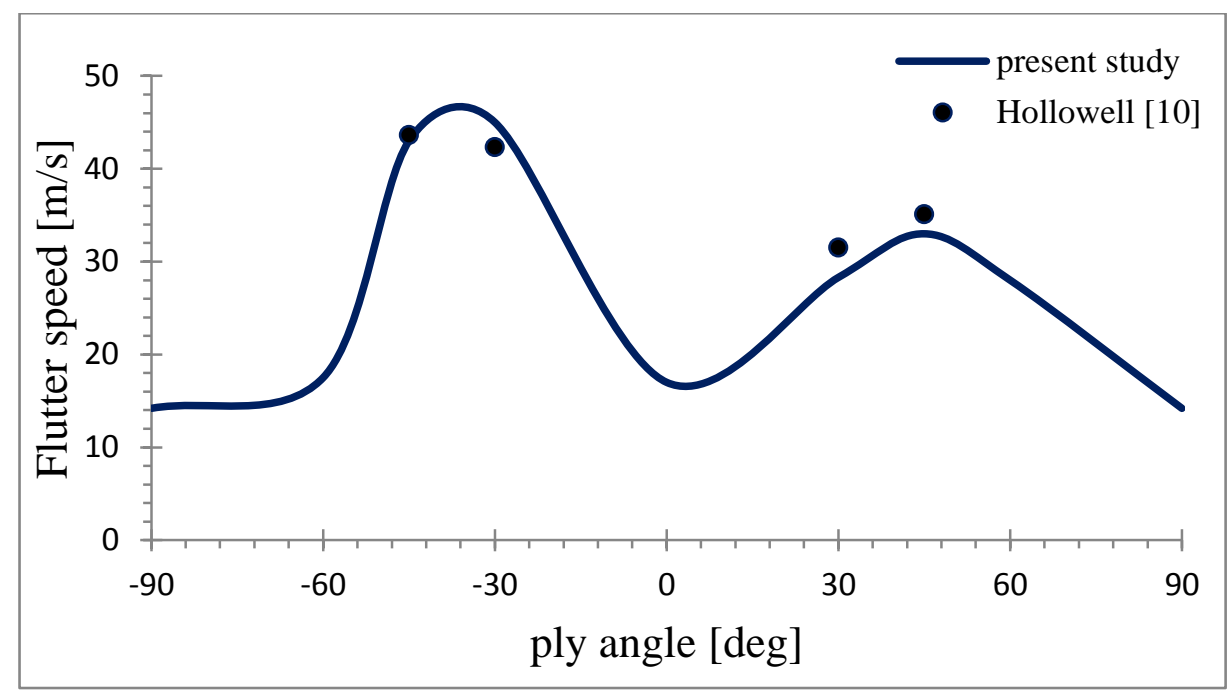

Fig (7) Variation of flutter speed with ply angle for a $\left[\theta_{2} / 0\right]_{\mathrm{s}}$ rectangle wing plate

Figure (8) illustrates variation of the flutter and divergence speeds using antisymmetric stacking sequence of layers $[\theta / 0 /-\theta]$. We can notice improvement of the flutter speed at all ply angles, especially at angle $\left(45^{\circ}\right)$, in addition to an increase in the divergence speed at negative angels (angles more than $90^{\circ}$ ). At positive angles $\left[20^{\circ}\right.$ up to $60^{\circ}$ ] there is complete suppression of divergence.

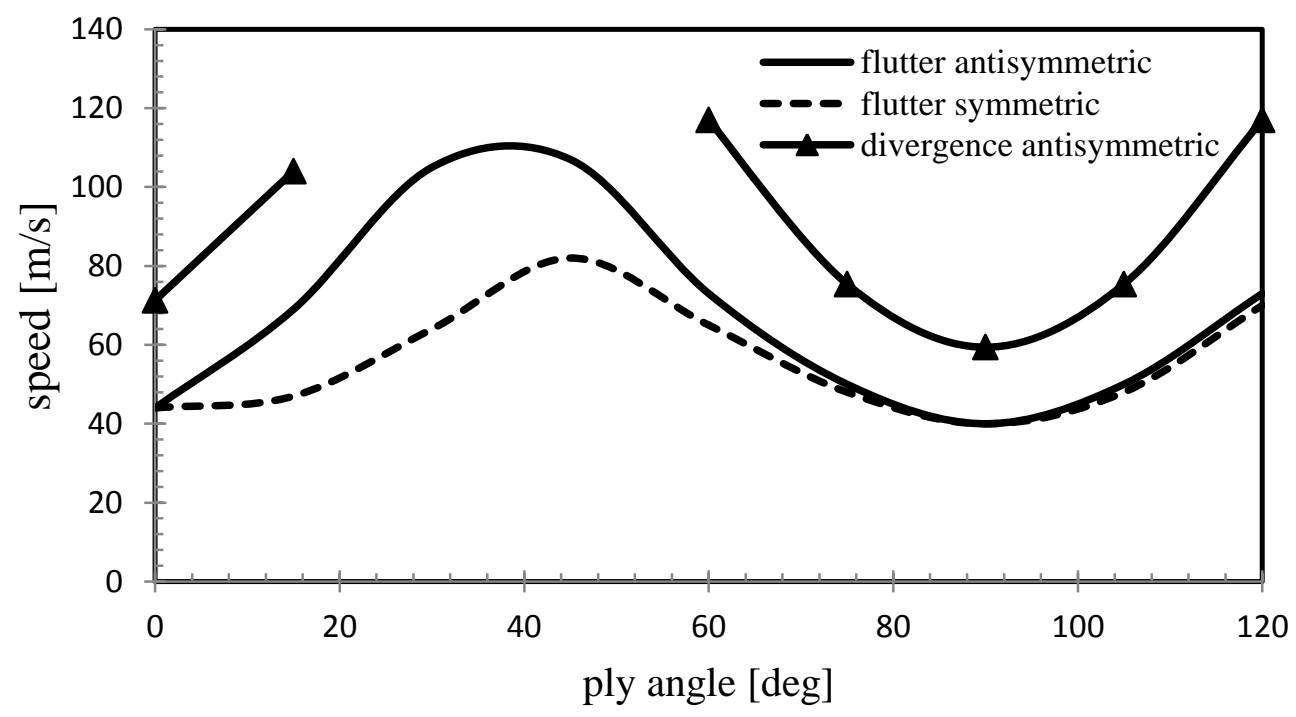

Fig (8) Variation of flutter and divergence speeds with ply angle for $[\theta / 0 /-\theta]$ rectangular plate wing

The relation between the natural frequencies and ply angle are shown in Fig (9) for symmetric and antisymmetric stacking sequences of layers. The natural frequencies for antisymmetric layers are lower than these for symmetric layers, except the second mode for angles between $10^{\circ}$ and $40^{\circ}$. 


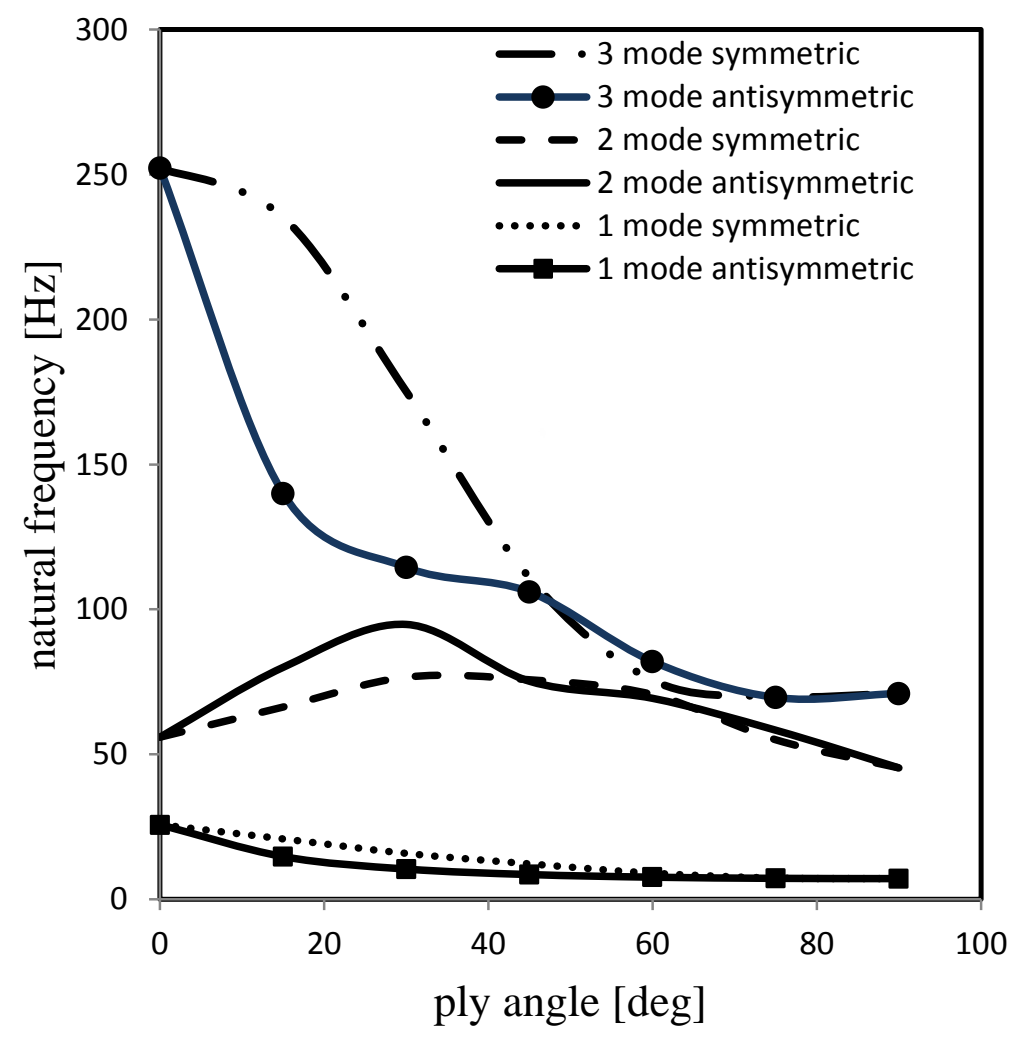

Fig (9) Variation of natural frequencies with ply angle for $[\theta / 0 /-\theta]$ rectangular plate wing

On the other hand, the flutter frequency for antisymmetric layup has greater values than for symmetric layup as shown in Fig (10).

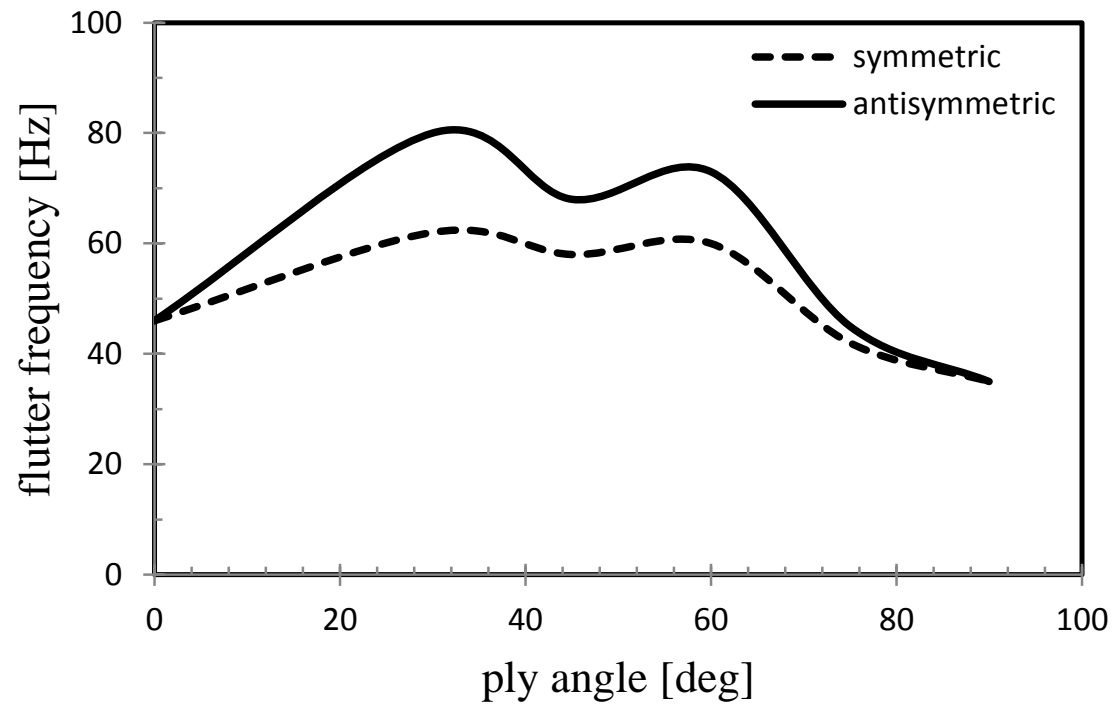

Fig (10) Variation of flutter frequency with ply angles for symmetric and antisymmetric wing 


\section{Conclusions}

Aeroelastic phenomena including divergence and flutter are investigated for unswept plate wing. Equivalent plate modeling for composite plate wing using Ritz function has been implemented. Steady and unsteady aerodynamic models have been used to calculate lift force and pressure distributions on the wing using the Doublet Point Method.

In harmonically oscillating motion, numerical calculations of spanwise lift distribution for rectangular plate and chordwise pressure distribution at plate root are made. The obtained results show good agreement with Morino results.

A suitable derivation for unsteady aerodynamic loads has been developed. Aeroelastic characteristics including divergence and flutter have been investigated for symmetric and antisymmetric stacking sequences of layers. The symmetric stacking sequence of layers is good for divergence at positive angles and bad for negative once. The flutter speed is higher for negative angles than for positive angles. In case of antisymmetric stacking sequence, both flutter and divergence speeds improve at all angles.

The flutter frequency for antisymmetric layup has higher values than for symmetric layup. The natural frequencies for antisymmetric stacking sequence are lower than for symmetric sequence, except for the second mode for angles between $10^{\circ}$ and $40^{\circ}$.

\section{References}

[1] Pong-Jeu Lu Kuo-Juin Lin, and Jiann-Quo Tarn, "Flutter Analysis of Cantilever Composite Plates in Subsonic Flow," AIAA Journal, vol. VOL. 27, NO. 8, pp. 11021109, 1989.

[2] T. Krishnamurthy and Brian H. Mason, "Equivalent Plate Analysis of Aircraft Wing with Discrete Source Damage," AIAA JOURNAL, vol. VA 23681, U.S.A., 2005.

[3] Y.C.Fung, "An Introduction to the Theory of aeroelasticity," Dover Publications, INC. New York, 1993.

[4] R.Bisplinghoff H. Ashly and R. L. Halfman, Ed., Aeroelasticity. Mineola, New York 11501: Dover publications inc,31East 2nd street, 1996, p.^pp. Pages.

[5] Eli Livne, "Equivalent Plate Structural Modeling for Wing Shape Optimization Including transverse Shear," AIAA Journal, vol. Vol. 32, No.6, pp. PP. 1278-1288, June 1994.

[6] Ulgen Gulcat, Ed., Fundementals Of Modern Unsteady Aerodynamics. Berlin Heidelberg: Springer, 2010, p.^pp. Pages.

[7] T. Ueda and E. H. Dowellf, "A New Solution Method for Lifting Surfaces in Subsonic Flow," AIAA Journal, pp. pp.348-355, 1982.

[8] Ching-Chiang Kuo Luigi Morino, "Subsonic Potential Aerodynamics for Complex Configurations: A General Theory," AIAA JOURNAL, vol. VOL. 12, NO. 2, pp. 191197, 1974.

[9] Wael G. Abdelrahman, "Static and Dynamic Behaviour of Composite Swept wings," Master M.Sc thesis, Department of aerospace Engineering Cairo University, Cairo

[10] John Dugundji Steven J. Hollwoell, "aeroelastic flutter and Divergence of stiffness Coupled, Graphite/Epoxy Cantilevered Plate," J. AIRCRAFT, vol. VOL. 21, NO. 1, pp. 69-76, 1984. 